\title{
ON DINI'S THEOREM AND A METRIC ON $C(X)$ TOPOLOGICALLY EQUTVALENT TO THE UNIFORM METRIC
}

\author{
GERALD BEER
}

\begin{abstract}
Let $X$ be a compact metric space and let $U C(X)$ denote the u.s.c. real valued functions on $X$. Let $\tau$ be a topology on $U C(X) . \Omega \subset U C(X)$ is called a Dini class of functions induced by $\tau$ if (1) $\Omega$ is $\tau$-closed, (2) $C(X) \subset \Omega$, (3) for each $h \in \Omega$ whenever $\left\{h_{n}\right\}$ is a decreasing sequence of u.s.c. functions convergent pointwise to $h$, then $\left\{h_{n}\right\} \tau$-converges to $h$. By Dini's theorem the topology of uniform convergence on $U C(X)$ induces $C(X)$ as its Dini class of functions. As a main result, when $X$ is locally connected we show that the hyperspace topology on $U C(X)$ obtained by identifying each u.s.c. function with the closure of its graph induces a larger Dini class of functions than $C(X)$, even though the restriction of this topology to $C(X)$ agrees with the topology of uniform convergence.
\end{abstract}

Let $X$ be a compact metric space and let $C(X)$ and $U C(X)$ denote the continuous and upper semicontinuous real valued functions on $X$, respectively. If $f \in C(X)$ and $\left\{h_{n}\right\}$ is a decreasing sequence of u.s.c. functions on $X$ convergent pointwise to $f$, then Dini's theorem [10] says that the convergence must be uniform. Moreover, if $\left\{f_{n}\right\}$ is a sequence in $C(X)$ convergent uniformly to an u.s.c. function $h$, then $h$ must be in $C(X)$. Thus, the class $C(X)$, viewed as a subclass of $U C(X)$, is induced by the topology of uniform convergence on $U C(X)$ in the following sense.

DEFINITION. Let $X$ be a compact metric space and let $\Omega \subset U C(X)$. If $\tau$ is a topology on $U C(X)$, then $\Omega$ is called a Dini class of functions induced by $\tau$ if

(1) $\Omega$ is $\tau$-closed,

(2) $C(X) \subset \Omega$,

(3) for each $h \in \Omega$ whenever $\left\{h_{n}\right\}$ is a decreasing sequence of u.s.c. functions convergent pointwise to $h$, then $\left\{h_{n}\right\} \tau$-converges to $h$.

It follows from the definition that $\tau$ can induce at most one Dini class. To see this, suppose that $\Omega$ and $\Omega^{\prime}$ are both Dini classes of $\tau$. Let $f \in \Omega$ be arbitrary. Since each u.s.c. function on $X$ is a pointwise limit of a decreasing sequence of continuous functions [6],

$$
f \in \operatorname{cl}_{\tau} C(X) \subset \operatorname{cl}_{\tau} \Omega^{\prime}=\Omega^{\prime} .
$$

Standard topologies on $U C(X)$ seem to induce nice Dini classes of functions. For example, let $\mu$ be a regular Borel measure on $X$. We first consider the convergence in measure topology on $U C(X)$. Recall that $\left\{h_{n}\right\}$ is said to converge in measure to

Received by the editors June 4, 1981 and, in revised form, November 11, 1981.

1980 Mathematics Subject Classification. Primary 26A15, 54B20, 54C35.

Key words and phrases. Semicontinuous function, Dini's theorem, Hausdorff metric . 
$h$ if for each $\epsilon>0$ there exists $N \in Z^{+}$such that for all $n \geq N$,

$$
\mu\left\{x:\left|h_{n}(x)-h(x)\right|>\epsilon\right\}<\epsilon .
$$

It is well known [7] that convergence in this sense is described by a pseudometric on the $\mu$-measurable functions on $X$ :

$$
\delta_{1}(f, g)=\int_{X} \frac{|f-g|}{1+|f-g|} d \mu .
$$

The $\delta_{1}$-topology induces $U C(X)$ as its Dini class. Conditions (1) and (2) hold trivially and condition (3) follows from Egoroff's theorem. In the same context define the $L_{1}(\mu)$ pseudometric $\delta_{2}: U C(X) \times U C(X) \rightarrow[0, \infty]$ by

$$
\delta_{2}(f, g)=\int_{X}|f-g| d \mu .
$$

We claim that $\delta_{2}$ induces $L_{1}(\mu) \cap U C(X)$, the u.s.c. functions that are $\mu$-integrable, as its Dini class. Condition (1) holds because $L_{1}(\mu)$ is closed under addition, condition (2) holds because $X$ is compact, and condition (3) follows from the Lebesgue dominated convergence theorem using as a majorant $g: X \rightarrow[0, \infty)$ defined by

$$
g(z)=\max \left\{\max _{x \in X} h_{1}(x),-\min \{h(z), 0\}\right\} .
$$

In this paper we shall focus our attention on two hyperspace topologies on $U C(X)$ considered in [2]. In short we identify members of $U C(X)$ with certain closed subsets of $X \times R$ and apply the Hausdorff metric. We review the essential features of this construction. Let $Y$ be a metric space with metric $\rho$ and for each $y$ in $Y$ let $B_{\lambda}[y]$ denote the closed ball of radius $\lambda$ about $y$. If $K$ is a closed subset of $Y$, the $\lambda$-parallel body of $K$, denoted by $B_{\lambda}[K]$, is the set $\bigcup_{y \in K} B_{\lambda}[y]$. If $C$ and $K$ are closed sets in $Y$ we define the Hausdorff distance of $C$ from $K$ to be

$$
D(C, K)=\inf \left\{\lambda: B_{\lambda}[K] \supset C \text { and } B_{\lambda}[C] \supset K\right\} .
$$

We refer the reader to Castaing and Valadier [3] or Nadler [9] for further information. Now let $X$ be a metric space with metric $d$ and make $X \times R$ a metric space using the metric $\rho$ defined by $\rho\left[\left(x_{1}, \alpha_{1}\right),\left(x_{2}, \alpha_{2}\right)\right]=\max \left\{d\left(x_{1}, x_{2}\right),\left|\alpha_{1}-\alpha_{2}\right|\right\}$. Since, in $X \times R$, closed and $\rho$-bounded sets are compact, the parallel body of each closed set will again be a closed set, a fact which we shall often use in the sequel. If $h$ and $g$ are in $U C(X)$ let $d_{1}(h, g)=\sup _{x \in X}|h(x)-g(x)|$. If $h$ is u.s.c., then the graph of $h$ will not in general be a closed set. Denote its closure in $X \times R$ by $\bar{h}$. If $h$ and $g$ are in $U C(X)$ denote the Hausdorff distance from $\bar{h}$ to $\bar{g}$ by $d_{2}(h, g)$. Although $d_{2}(h, g)=\infty$ is a possibility, $d_{2}$ is an extended valued metric, not just a pseudometric. Next, if $h$ is u.s.c. its hypograph $\{(x, \alpha): \alpha \leq h(x)\}$ will be symbolized by hypo $h$. It is well known that u.s.c. functions are characterized as those having closed hypographs. If $h$ and $g$ are in $U C(X)$ denote the Hausdorff distance between their hypographs by $d_{3}(h, g)$. Surprisingly, $d_{3}$ is a finite valued metric on $U C(X)$ even though $h$ and $g$ may be unbounded.

We shall need the following facts established in [2] which we state as theorems.

THEOREM A. If $h$ and $g$ are in $U C(X)$ then $d_{1}(h, g) \geq d_{2}(h, g) \geq d_{3}(h, g)$. 
THEOREM B. The metrics $d_{1}$ and $d_{2}$, when restricted to $C(X)$, define the same topology.

THEOREM C. Let $\left\{h_{n}\right\}$ be a sequence of u.s.c. functions on $X$. Then $\left\{h_{n}\right\}$ converges to $h$ in the metric $d_{3}$ if and only if

(1) for each $x$ in $X$ whenever $\left\{x_{n}\right\} \rightarrow x$ then $\lim \sup _{n \rightarrow \infty} h_{n}\left(x_{n}\right) \leq h(x)$,

(2) for each $x$ in $X$ there exists a sequence $\left\{x_{n}\right\}$ convergent to $x$ for which $\lim _{n \rightarrow \infty} h_{n}\left(x_{n}\right)=h(x)$.

In view of Theorem $\mathrm{C}$ above, first proved by Mosco for convex functions [8], $d_{3}$-convergence is equivalent to the notion of infimal convergence for lower semicontinuous functions introduced by Wijsman [11] (but only in the context of compact spaces). Infimal convergence was then studied in a more general setting by DeGiorgi and Franzoni [4], and has received recent attention from Dolecki, Salinetti, and Wets [5] where it is now called epi-convergence.

We consider the metric $d_{3}$ first. Since this metric is in some sense a Baire category dual to the metric $\delta_{1}[1]$, one expects that the Dini class induced by $d_{3}$ would also be all of $U C(X)$. This conjecture is correct. We need only verify condition (3) in the definition. To this end let $\left\{h_{n}\right\}$ be a decreasing sequence of u.s.c. functions convergent pointwise to an u.s.c. function $h$. Fix $x$ in $X$ and let $\epsilon$ be positive. Choose $N$ so large that $h_{N}(x)<h(x)+\frac{\epsilon}{2}$ and $\delta>0$ such that if $d(y, x)<\delta$ then $h_{N}(y)<h_{N}(x)+\frac{\epsilon}{2}$. Clearly if $d(y, x)<\delta$ and $n \geq N$ we have $h_{n}(y)<h(x)+\epsilon$. This implies that whenever $\left\{x_{n}\right\} \rightarrow x$ we obtain $\lim _{\sup _{n \rightarrow \infty}} h_{n}\left(x_{n}\right)<h(x)+\epsilon$. Condition (1) of Theorem $\mathrm{C}$ now follows. Condition (2) of Theorem $\mathrm{C}$ is already satisfied, for $\lim _{n \rightarrow \infty} h_{n}(x)=h(x)$.

Since $d_{1}$ and $d_{2}$, when restricted to $C(X)$, are topologically equivalent, one would suppose that they induce the same Dini class. This is not the case: $d_{2}$ induces a larger one than $C(X)$ provided that $X$ is locally connected. To properly describe this class we need some preliminary material.

Let $h$ be a real valued function on $X$, not necessarily u.s.c. For each $x \in X$ let

$$
L(h, x)=\left\{\alpha: \alpha \in R \text { and } \exists\left\{x_{n}\right\} \rightarrow x \text { for which }\left\{h\left(x_{n}\right)\right\} \rightarrow \alpha\right\} .
$$

Notice that $h(x)$ is an element of $L(h, x)$; it is the largest (resp. smallest) element of $L(h, x)$ if $h$ is u.s.c. (resp. l.s.c.) at $x$. For each $n \in Z^{+}$let

$$
L_{n}(h, x)=\operatorname{cl}\left\{h(y): d(x, y) \leq \frac{1}{n}\right\}=\operatorname{cl} h\left(B_{1 / n}[x]\right) .
$$

We leave the proof of the following simple lemma to the reader.

LEMMA 1. Let $X$ be a compact metric space and let $h: X \rightarrow R$. For each $x \in X$, $\bigcap_{n=1}^{\infty} L_{n}(h, x)=\{\alpha:(x, \alpha) \in \bar{h}\}=L(h, x)$.

As a result of Lemma $1, L(h, x)$ is a closed set. It is possible that each set $L(h, x)$ can be bounded without $h$ itself being bounded, e.g., define $h:[0,1] \rightarrow R$ by $h(0)=0$ and $h(x)=\frac{1}{x}$ otherwise.

LEMMA 2. Let $X$ be a compact metric space and let $h: X \rightarrow R$. Suppose for each $x \in X$ the set $L(h, x)$ is bounded. The following are equivalent.

(i) $h$ is bounded.

(ii) For each $x,\left\{L_{n}(h, x)\right\}$ converges to $L(h, x)$ in the Hausdorff metric.

(iii) The multifunction $\Gamma$ on $X$ defined by $\Gamma(x)=L(h, x)$ is upper semicontinuous. 
ProOF. (i) $\rightarrow$ (ii) Fix $x$ in $X$. Since for each $n, L_{n}(h, x) \supset L(h, x)$, we need only show that given $\epsilon>0$ the parallel body $B_{\epsilon}[L(h, x)]$ contains $\left\{L_{n}(h, x)\right\}$ eventually. If not, since $B_{\epsilon}[L(h, x)]$ is closed and $\left\{L_{n}(h, x)\right\}$ is a decreasing sequence of sets, we can find, for each $n$, a point $x_{n}$ satisfying $d\left(x_{n}, x\right) \leq \frac{1}{n}$ but $h\left(x_{n}\right) \notin B_{\epsilon}[L(h, x)]$. By hypothesis $\left\{h\left(x_{n}\right)\right\}$ is bounded; so, it has a convergent subsequence. The limit cannot belong to $L(h, x)$, for $L(h, x) \subset$ int $B_{\epsilon}[L(h, x)]$. This violates the definition of $L(h, x)$.

(ii) $\rightarrow$ (iii) Let $x$ be arbitrary and let $V$ be an open set in $R$ containing $\Gamma(x)$. We must produce a neighborhood $U$ of $x$ such that if $y \in U$ then $\Gamma(y) \subset V$. Since $\Gamma(x)=L(h, x)$ is compact, there exists $\epsilon>0$ such that $B_{\epsilon}[L(h, x)] \subset V$. Choose $n$ so large that $L_{n}(h, x) \subset B_{\epsilon}[L(h, x)]$. If $d(x, y)<\frac{1}{n}$ and $\frac{1}{m}<\frac{1}{n}-d(x, y)$ then

$$
\Gamma(y)=L(h, y) \subset L_{m}(h, y) \subset L_{n}(h, x) \subset B_{\epsilon}[\Gamma(x)] \subset V .
$$

(iii) $\rightarrow$ (i) Suppose $h$ fails to be bounded. Since $X$ is compact we can find a convergent sequence $\left\{x_{n}\right\}$ in $X$ such that $\lim _{n \rightarrow \infty}\left|h\left(x_{n}\right)\right|=\infty$. Set $x=\lim _{n \rightarrow \infty} x_{n}$. Clearly, $\left\{h\left(x_{n}\right)\right\}$ cannot be in the bounded set $B_{\epsilon}[L(h, x)]$ eventually. Since $B_{\epsilon}[L(h, x)]$ is a neighborhood of $L(h, x)$ and for each $n, h\left(x_{n}\right) \in \Gamma\left(x_{n}\right)$, the multifunction $\Gamma$ fails to be u.s.c. at $x$.

Next let $\Omega=\{h: h$ is u.s.c. and bounded and $\forall x, L(h, x)$ is convex $\}$. Notice that the inclusion $\Omega \supset C(X)$ is proper. For example $f:[0,1] \rightarrow R$ defined by

$$
f(x)= \begin{cases}\sin \frac{1}{x} & \text { if } x \neq 0 \\ 1 & \text { if } x=0\end{cases}
$$

is in $\Omega-C(X)$. If $h \in \Omega$ then each set $L(h, x)$ is precisely the closed line segment [liminf $\operatorname{lix}_{y \rightarrow x} h(y), h(x)$ ]. Finally, $\Omega$ may be described as the class of u.s.c. functions for which the multifunction $\Gamma$ of Lemma 2 is both compact convex valued and u.s.c.

LEMMA 3. $\Omega=\{h: h$ is bounded and u.s.c. and $\bar{h} \supset$ hypo $h-\operatorname{int}($ hypo $h)\}$.

Proof. Let $h$ be bounded and u.s.c. It is easy to see that $(x, \alpha) \in$ hypo $h-$ int(hypo $h$ ) if and only if $\liminf _{y \rightarrow x} h(y) \leq \alpha \leq h(x)$. If hypo $h-\operatorname{int}($ hypo $h) \subset$ $\bar{h}$, then for each $x$, Lemma 1 implies that the segment [liminf $\left.\operatorname{in}_{y \rightarrow x} h(y), h(x)\right] \subset$ $L(h, x)$. Since the other inclusion always holds whenever $h$ is u.s.c., we obtain $h \in$ $\Omega$. Conversely, if $h \in \Omega$ then for each $x, L(h, x)=\left[\liminf _{y \rightarrow x} h(y), h(x)\right]$ so that if $(x, \alpha)$ lies in hypo $h$ - int(hypo $h)$, it must also lie in $\bar{h}$ by Lemma 1 .

Local connectivity of $X$ is not required to establish condition (3) for $\Omega$ to be a $d_{2}$-induced Dini class of functions. Before we can prove this we need one more lemma.

LEMMA 4. Let $X$ be a compact metric space and let $\left\{h_{n}\right\}$ be a sequence in $U C(X) d_{3}$-convergent to a bounded u.s.c. function $h$. Then for each $\lambda>0$ there exists $N \in Z^{+}$such that $n \geq N$ implies $\bar{h} \subset B_{\lambda}\left[\bar{h}_{n}\right]$.

PROOF. If not, there exists $\lambda$ and a subsequence of $\left\{h_{n}\right\}$ such that $\bar{h}$ fails to lie in the $\lambda$-parallel body of the closure of the graph of each term of the subsequence. By relabeling we can assume that, for each $n, \bar{h} \not \subset B_{\lambda}\left[\bar{h}_{n}\right]$. Since $B_{\lambda}\left[\bar{h}_{n}\right]$ is a closed set, for each $n$ we can find $\left(x_{n}, h\left(x_{n}\right)\right)$ in $\bar{h}-B_{\lambda}\left[\bar{h}_{n}\right]$. Once again passing to a subsequence we can assume that $\left\{\left(x_{n}, h\left(x_{n}\right)\right)\right\}$ is convergent and is therefore Cauchy. Choose $N$ so large that $m>n \geq N$ implies that $\rho\left[\left(x_{n}, h\left(x_{n}\right)\right),\left(x_{m}, h\left(x_{m}\right)\right)\right]<\frac{\lambda}{2}$. By the second condition in Theorem $C$ there exists $K \geq N$ and a point in $\bar{h}_{K}$ that 
is within $\frac{\lambda}{2}$ of $\left(x_{N}, h\left(x_{N}\right)\right)$. It follows that $\left(x_{K}, h\left(x_{K}\right)\right)$ has distance at most $\lambda$ from $\bar{h}_{K}$, a contradiction.

We note that if $\left\{h_{n}\right\}$ is a sequence of bounded u.s.c. functions $d_{3}$-convergent to $h$, then $\left\{h_{n}\right\}$ "half- $d_{2}$ converges" to $h$ in the above sense if and only if $h$ is bounded.

THEOREM 1. Let $X$ be a compact metric space with metric $d$. Let $h \in \Omega$ and let $\left\{h_{n}\right\}$ be a decreasing sequence of u.s.c. functions convergent pointwise to $h$. Then $\left\{h_{n}\right\} d_{2}$-converges to $h$.

ProOF. Let $\lambda$ be positive and set $E_{\lambda}=\bigcup_{x \in X} B_{\lambda}[\{(x, \alpha): \alpha \in L(h, x)\}]$. We claim that if $\epsilon$ is sufficiently small then $B_{\epsilon}$ [hypo $\left.h\right]$-hypo $h \subset E_{\lambda}$. If not we can find for each $n \in Z^{+}$a point $\left(x_{n}, \alpha_{n}\right)$ such that $\alpha_{n}>h\left(x_{n}\right),\left(x_{n}, \alpha_{n}\right) \in B_{1 / n}$ [hypo h], but $\left(x_{n}, \alpha_{n}\right) \notin E_{\lambda}$. By passing to a subsequence we can assume $\left\{x_{n}\right\}$ converges to some point $x$. Since hypo $h$ is a closed set and $\bigcap_{n=1}^{\infty} B_{1 / n}[$ hypo $h]=$ hypo $h$, it is easy to see that $\lim \sup _{n \rightarrow \infty} \alpha_{n} \leq h(x)$. Set $\alpha=\limsup _{n \rightarrow \infty} \alpha_{n}$. Since $h \in \Omega$ and

$$
\alpha \geq \liminf _{n \rightarrow \infty} h\left(x_{n}\right) \geq \liminf _{y \rightarrow x} h(y)
$$

we have $\alpha \in L(h, x)$. There exists $n \in Z^{+}$such that $d\left(x_{n}, x\right) \leq \lambda$ and $\left|\alpha_{n}-\alpha\right| \leq \lambda$. However, this implies $\left(x_{n}, \alpha_{n}\right) \in E_{\lambda}$, a contradiction. This establishes the claim.

Next, choose a fixed $\epsilon$ for which $B_{\epsilon}[$ hypo $h]-$ hypo $h \subset E_{\lambda}$. Since $\left\{h_{n}\right\}$ $d_{3}$-converges to $h$, there exists $N \in Z^{+}$such that $n \geq N$ implies hypo $h_{n} \subset$ $B_{\epsilon}[$ hypo $h]$. As a consequence since $B_{\epsilon}[$ hypo $h]$ is closed, $\bar{h}_{n} \subset B_{\epsilon}[$ hypo $h]$ for each such $n$. Since each $h_{n}$ majorizes $h$, for all $x$, either $\left(x, h_{n}(x)\right) \in \bar{h}$ or $\left(x, h_{n}(x)\right) \in$ $B_{\epsilon}[$ hypo $h]-$ hypo $h$. In the first case $\left(x, h_{n}(x)\right)$ has distance zero from $\bar{h}$. In the second case, by the definition of $E_{\lambda},\left(x, h_{n}(x)\right)$ has distance at most $\lambda$ from some point $(x, \alpha)$ where $\alpha \in L(h, x)$. By Lemma $1,(x, \alpha) \in \bar{h}$; so $\left(x, h_{n}(x)\right)$ has distance at most $\lambda$ from $\bar{h}$. Since $x$ was arbitrary, it follows that, for each $n \geq N, \bar{h}_{n} \subset B_{\lambda}[\bar{h}]$. The inclusion $B_{\lambda}\left[\bar{h}_{n}\right] \supset \bar{h}$ holds for all $n$ sufficiently large by Lemma 4 .

The next theorem, which shows that $\Omega$ is $d_{2}$-closed, completes the demonstration that $\Omega$ is a Dini class of functions induced by $d_{2}$. As the next example shows, the hypothesis that $X$ be locally connected cannot be omitted.

EXAMPLE. Let $X=\left\{0,1, \frac{1}{2}, \frac{1}{3}, \ldots\right\}$ considered as a subspace of the line. Let $h=\chi_{\{0\}}$ and for each $n$ let $h_{n}=\chi_{\{0\} \cup\{t: k \geq n\}}$. Then $\left\{h_{n}\right\}$ is a decreasing sequence of continuous functions $d_{2}$-convergent to $h$. However, since $L(h, 0)=$ $\{0,1\}$, a nonconvex set, $h$ fails to be in $\Omega$.

THEOREM 2. Let $X$ be a compact locally connected metric space. Let $\left\{h_{n}\right\}$ be a sequence in $\Omega, d_{2}$-convergent to an u.s.c. function $h$. Then $h \in \Omega$.

ProOF. By the remark following Lemma $4, h$ is bounded. Fix $x$ in $X$. If $h$ is continuous at $x$, then $L(h, x)$ is convex. Otherwise, choose $\alpha$ strictly between $\liminf _{y \rightarrow x} h(y)$ and $h(x)$. By Lemma 1 it suffices to show that $(x, \alpha) \in \bar{h}$. Choose $\epsilon<\alpha-\liminf _{y \rightarrow x} h(y)$ and let $C$ be a connected neighborhood of $x$ in $B_{\epsilon / 2}[x]$. Next select $\delta>0$ such that $B_{\delta}[x] \subset C$. There exists $N \in Z^{+}$such that $n \geq N$ implies $d_{2}\left(h_{n}, h\right)<\delta$. By Theorem A, $d_{3}\left(h_{n}, h\right) \leq d_{2}\left(h_{n}, h\right)$; so, for each $n \geq N$ we have $B_{\delta}[(x, \alpha)] \cap$ hypo $h_{n} \neq \emptyset$. Observing that $B_{\delta}[(x, \alpha)] \subset C \times[\alpha-\delta, \alpha+\delta]$ we distinguish two mutually exclusive cases:

(1) $\exists n \geq N$ for which $(C \times[\alpha-\delta, \alpha+\delta]) \cap\left(\right.$ hypo $h_{n}-\operatorname{int}\left(\right.$ hypo $\left.\left.h_{n}\right)\right) \neq \emptyset$.

(2) $\forall n \geq N(C \times[\alpha-\delta, \alpha+\delta]) \cap$ hypo $h_{n} \subset \operatorname{int}\left(\right.$ hypo $\left.h_{n}\right)$. 
Case (1). For such an integer $n$ select $(y, \beta)$ in $(C \times[\alpha-\delta, \alpha+\delta]) \cap$ (hypo $h_{n}-$ $\operatorname{int}\left(\right.$ hypo $\left.\left.h_{n}\right)\right)$. Since $h_{n} \in \Omega$, Lemma 3 says that $(y, \beta) \in \bar{h}_{n}$. Since $d_{2}\left(h, h_{n}\right) \leq$ $\frac{\epsilon}{2}$ there exists $(w, \theta) \in \bar{h}$ with $\rho$-distance from $(y, \beta)$ at most $\frac{\epsilon}{2}$. It follows that $\rho[(w, \theta),(x, \alpha)] \leq \epsilon$. Thus, $B_{\epsilon}[(x, \alpha)]$ meets $\bar{h}$, and since $\bar{h}$ is a closed set, we conclude that $(x, \alpha) \in \bar{h}$.

Case (2). By the choice of $N$, for each $n \geq N$, we can find $x_{n}$ such that $\rho\left[\left(x_{n}, h_{n}\left(x_{n}\right)\right),(x, h(x))\right] \leq \delta$. Clearly $h_{n}\left(x_{n}\right) \geq \alpha-\delta$; so, $\left(x_{n}, \alpha-\delta\right)$ lies in hypo $h_{n}$. By assumption, for each such $n$ we have

$$
\{(w, \alpha-\delta): w \in C\} \cap\left(\text { hypo } h_{n}-\operatorname{int}\left(\text { hypo } h_{n}\right)\right)=\emptyset \text {. }
$$

Thus the relatively open subsets of $C$,

$$
\begin{gathered}
E_{n}=\left\{w: w \in C \text { and }(w, \alpha-\delta) \in \operatorname{int}\left(\text { hypo } h_{n}\right)\right\}, \\
F_{n}=\left\{w: w \in C \text { and }(w, \alpha-\delta) \notin \text { hypo } h_{n}\right\},
\end{gathered}
$$

partition the connected set $C$. Since for each $n \geq N, x_{n} \in E_{n}$, we conclude that $E_{n}=C$ for all such $n$. As a result, for each $w \in C$ and each such $n$, we have $h_{n}(w)>\alpha-\delta \geq \alpha-\frac{\epsilon}{2}>\liminf _{y \rightarrow x} h(y)+\frac{\epsilon}{2}$. Choose $z \in X$ for which $\rho\left[(z, h(x)),\left(x, \liminf _{y \rightarrow x} h(y)\right)\right] \leq \frac{\delta}{2}$. By Theorem C, if $n \geq N$ is chosen sufficiently large, then we can find $\left(w, h_{n}(w)\right)$ whose $\rho$-distance from $(z, h(z))$ is at most $\frac{\delta}{2}$. It follows that $w \in C$ and $h_{n}(w) \leq \liminf _{y \rightarrow x} h(y)+\frac{\epsilon}{2}$. This is a contradiction so that Case (2) cannot occur. Hence, we revert to Case (1) and the theorem is proved.

\section{BIBLIOGRAPHY}

1. G. Beer, $A$ natural topology for upper semicontinuous functions and a Baire category dual for convergence in measure, Pacific J. Math. 96 (1981), 251-263.

2. $\ldots$, Upper semicontinuous functions and the Stone approximation theorem, J. Approximation Theory 34 (1982), 1-11.

3. C. Castaing and M. Valadier, Convex analysis and measurable multifunctions, Springer-Verlag, Berlin and New York, 1977.

4. E. DeGiorgi and T. Franzoni, Su un tipo di convergenza variazionale, Atti Accad. Naz. Lincei Rend. Cl. Sci. Fis. Mat. Natur. (8) 58 (1975), 842-850.

5. S. Dolecki, G. Salinetti and R. Wets, Convergence of functions: equi-semicontinuity, Proc. London Math. Soc. (to appear).

6. R. Engleking, General topology, Polish Scientific Publishers, Warsaw, 1977.

7. E. Hewitt and C. Stromberg, Real and abstract analysis, Springer-Verlag, Berlin and New York, 1965.

8. U. Mosco, Convergence of convex sets and the solutions of variational inequalities, Adv. in Math. 3 (1969), 510-585.

9. S. Nadler, Hyperspaces of sets, Dekker, New York, 1978.

10. R. Royden, Real analysis, Macmillan, New York, 1968.

11. R. Wijsman, Convergence of sequences of convex sets, cones, and functions. II, Trans. Amer. Math. Soc. 123 (1966), 32-45.

Department of Mathematics, California State University, Los Angeles, CALIFORNIA 90032 